\title{
Christine Windbichler zum 70. Geburtstag
}

CHRISTIAN ARMBRÜSTER

GREGOR BACHMANN

THEODOR BAUMS

WALTER BAYER

FRANK BAYREUTHER

BURKHARD BOEMKE

CHRISTOPH BRÖMMELMEYER

RICHARD M. BUXBAUM

MATTHIAS CASPER

GERHARD DANNEMANN

MEINRAD DREHER

KATHARINA DE LA DURANTAYE

JENS EKKENGA

CHRISTOPH ENGEL

ANDREAS ENGERT

LORENZ FASTRICH

ANDREAS MARTIN FLECKNER

HOLGER FLEISCHER

AXEL FLESSNER

TIM FLORSTEDT

MARTIN FRANZEN

INGA FROHMANN

CARsten Gerner-Beuerle

STEFAN GRUNDMANN

BARBARA GRUNEWALD

MATHIAS HABERSACK

SILVIO HÄNSENBERGER

LUTZ HAERTLEIN

JOHANNES HAGER

KRISTINA HARRER-KOULIEV

FELIX HARTMANN

MARTIN HENSSLER

HERIBERT HIRTE

PETER HOMMELHOFF

KLAUS J. HOPT

MATTHIAS JACOBS
SUSANNE KALSS

JENS KOCH

MiCHAEL KORT

RÜDIGER KRAUSE

GERD KRIEGER

KASPAR KROLOP

KATJA LANGENBUCHER

THOMAS LOBINGER

LINA LUYKEN

ANJA MENGEL

HANNO MERKT

RALF MICHAELS

HANNAH MODI

SVEN MÖLLER

FLORIAN MÖSLEIN

HANS-FRIEDRICH MÜLLER

ANDREAS NELLE

ULRICH NOACK

EVA INÉS OBERGFELL

HARTMUT OETKER

Christoph G. Paulus

HERMANN REICHOLD

REINHARD RICHARDI

KARL RIESENHUBER

MARKUS ROTH

GIESELA RÜHL

FRANZ JÜRGEN SÄCKER

CARSTEN SCHÄFER

JAN-ERIK SCHIRMER

KARSTEN SCHMIDT

KLAUS UlRiCH SCHMOLKE

WOLFGANG SCHÖN

Claudia Schubert

PETER SCHÜREN

JOACHIM SCHULZE-OSTERLOH

ULRICH SEIBERT 
WOLFGANG SERVATIUS

REINHARD SINGER

GERALD SPINDLER

THOMAS STAPPERFEND

GAABRIEL TAVITS

CHRISTOPH TEICHMANN

JAN THIESSEN

TOBIAS TRÖGER

EBERHARD VETTER

GERHARD WAGNER

GABRIELA V. WALLENBERG

ARTUR WANDTKE
MARTIN WEBER

MANFRED WEISS

AXEL V. WERDER

HARM PETER WeSTERMANN

HERBERT WiEDEMANN

ISABELLE WILDHABER

JAAP WINTER

ROLAND WOLF

JOACHIM WUTTE

MiKo YeBOAH-SMITH

DIRK ZETZSCHE 